\title{
New organometallic reagents for olefin synthesis
}

\author{
Jeffrey Schwartz*, Georgia M. Arvanitis, John A. Smegel, Ingrid K. Meier, \\ Susan M. Clift and Donna Van Engen \\ Chemistry Department, Princeton University, Princeton, NJ 08544 USA
}

\begin{abstract}
The synthesis, structural characterization, and reactivity of zirconium metalloazines are presented. A mechanism for olefination of aldehydes or ketones by metalloazines is discussed based on a series of mechanistic studies concerning this reaction.
\end{abstract}

\section{INTRODUCTION}

To take advantage of reactivity of transition metal complexes in order to develop new systems for use in organic synthesis is a topic of considerable interest in modern organic chemistry. We are studying the creation of methodology which complements olefin synthesis by the "classical" Wittig reaction, and we have focused our attention on developing procedures which obviate the formation of by-products (such as triorganophospine oxides) which are not only expensive to discard or recycle, but also which present problems with regard to separation from reaction mixtures.

\section{CARBENE COMPLEXES}

Our first approach to the development of olefination reagents was based on observations originally made for the "Tebbe" reagent that low-valent titanium methylene complexes could methylenate $a$ wide variety of organic carbonyls under mild conditions ${ }^{1}$. Several tantalum ${ }^{2}$ and tungsten ${ }^{3}$ alkylidene analogs have also been prepared. None of these reagent systems are, however, without drawbacks. The Tebbe reagent is easy to make, but its chemistry is limited to methylene transfer. In the nlobium and tungsten cases, the starting materials are not easily accessible to synthetically oriented organic chemists. In every case metallic by-products have not been shown to be recyclable, and control of $E, Z$ stereochemistry has not yet been demonstrated. Our first efforts involved the synthesis of long alkyl chain anaologs of the Tebbe reagent: $C p_{2} \mathrm{Zr}=\mathrm{C}(\mathrm{H}) \mathrm{R}$. These compounds could be made from an acetylene via a tedious synthesis involving conversion of the acetylene to a metal alkenyl and thence to a heterobimetallic alkylidene-bridged complex ${ }^{4}$ which could be selectively cleaved to give the carbene complex ${ }^{5}$. We were able to demonstrate that these unsymmetrically substituted carbene complexes could react with imine or imidate derivatives of a carbonyl group containing material, and through variation in the size of the organic group on nitrogen, the $E, Z$ stereochemistry of olefination could be affected ${ }^{6}$. However, the tedious synthesis of the zirconium carbene complexes precludes this approach as a viable method from the point of view of practical organic synthesis.

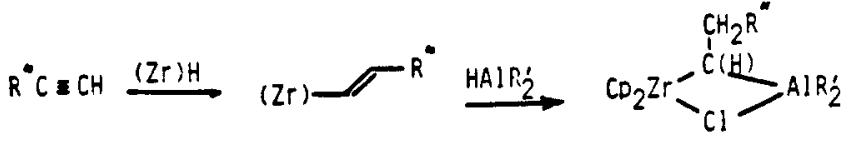

$$
\begin{aligned}
& =<_{\mathrm{CH}_{2} \mathrm{R}^{\prime \prime}}^{\mathrm{H}} \stackrel{\mathrm{R}}{\longleftarrow} \mathrm{CN}=\left\langle\quad \mathrm{CD}_{2} \mathrm{Zr}=\mathrm{C}(\mathrm{H}) \mathrm{CH}_{2} \mathrm{R}^{\prime \prime}\right.
\end{aligned}
$$

\section{METALLOAZINES}

We next considered the possibility that transition metal derivatives could be prepared from readily available organic starting materials through procedures familiar to synthesisoriented organic chemists. Our intent was to create a family of compounds $M-X-C(H) R$, in which the $[-\mathrm{X}-\mathrm{C}(\mathrm{H}) \mathrm{R}]$ group could be obtained in straightforward fashion and in which the reactivity of the $[X-C(H) R]$ group paralleled that of its carbene complex analogs, $M=C(H) R$. 
After forming a metal complex, the $X$ group should be easily lost in a condensation reaction with an olefination reagent. In this context we developed the chemistry of "metalloazines", species which can be obtained from hydrazones or from hydrazone derivatives, and from which dinitrogen can be extruded in an olefination process.

\section{Electrophilic metalloazines: molybdenum complexes (ref. 7)}

Molybdenum metalloazines can be formed by reaction between low-valent molybdenum complexes and diazolkanes which are obtained by partial oxidation of hydrazones. In this reaction, the molybdenum center undergoes a formal oxidative addition of the diazo unit. Reactivity of metalloazines can be anticipated by consideration of resonance structures for them. Because of predicted relative electronegativities of molybdenum(IV) and carbon, the resonance form indicating electrophilicity at the carbon unit seems most important, In fact molybdenum metalloazines behave as reactivity analogs of the carbonyl compounds from which they were derived; specifically, these metalloazines react with phosphoranes to generate olefins. In contrast to the "Wittig" reaction, however, which yields phosphine oxide in the "redox" reaction carbonyl $\rightarrow$ olefin, the reaction between the molybdenum metalloazine and the phosphorane yields the reduced molybdenum starting material, the free phosphine, the olefin, and dinitrogen. In this process, therefore, "reduction" of the carbonyl unit to the olefin is balanced by stepwise oxidation of hydrazine to dinitrogen. Dinitrogen and water are the only by-products; all other materials can be recovered and reused directly.

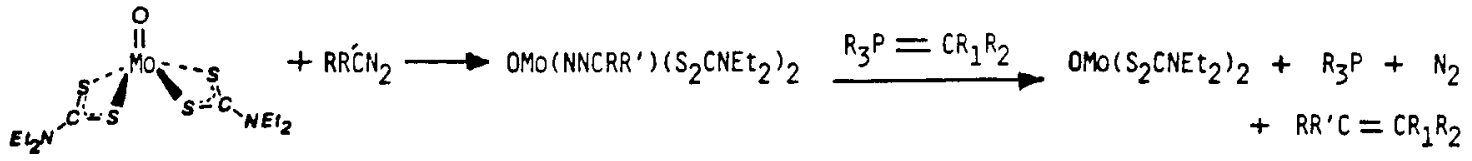

\section{Nucleophilic metalloazine: zirconium complexes (ref. 8)}

Reduced-valent zirconiun compounds also condense with diazoalkanes to yield zirconium metalloazines, species which have been found to be dimeric. Because of anticipated relative electronegativities of zirconium and carbon, that resonance structure showing negative charge build-up on carbon is now predicted to be important. Thus an appealing aspect of the use of transition metal derivatives in organic synthesis is made readily apparent by these two examples: beginning with a common starting material, for example a hydrazone derivative, by condensation with a relatively electronegative metal complex center (molybdenum) or a relatively electropositive one (zirconium), simple adducts can be obtained (the metalloazine) which can show "opposite" reactivity at the carbonyl-derived carbon center.

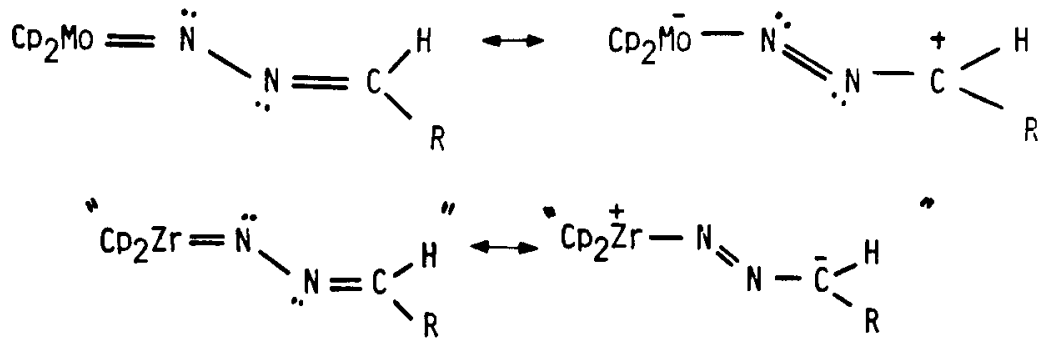

We have also found 8 that hydrazones, themselves, can condense with commercially available, easy-to-handle zirconocene dichloride in the presence of base cu yieic lite same metalloazines obtained in the oxidative addition process noted above. This procedure is obviously superior to that one based on diazoalkanes because it uses the hydrazone directly without requiring initial partial oxidation. Because of the unusual "umpolung" of reactivity 9 of the carbonyl-derived carbon center, the chemistry of zirconium metalloazines now commands our greatest attention.

$\mathrm{X}$-ray crystallographic analysis ${ }^{8}$ of several $z$ irconium metalloazines reveals a dimeric structure, and in these materials the metalloazine units are involved in unsymmetrical bridging with regard to the zirconium centers; the $\mathrm{Zr}-\mathrm{N}$ bonds are relatively unhindered. Room temperature proton and carbon NMR analysis of zirconium metalloazines suggests a symmetrical structure, but spectra obtained at low temperature are consistent with the $\mathrm{X}$-ray derived structure. Thus the metalloazines are fluxional with regard to the mode of nitrogen group coordination to the zirconium centers.

Zirconium metalloazines illustrate "umpolung" of reactivity at the carbonyl-derived carbon center in their condensation reactions with aldehydes or ketones. ${ }^{8}$ This reaction, which formally constitutes the unsymetrical coupling of two carbonyl groups, yields olefin, dinitrogen, and a crystalline zirconium oxo-polymer which can be removed easily from the reaction mixture and recycled to the starting zirconocene dichloride with anhydrous hydrogen chloride. Thus, in this coupling reaction, the "reduction" of the two carbonyl 

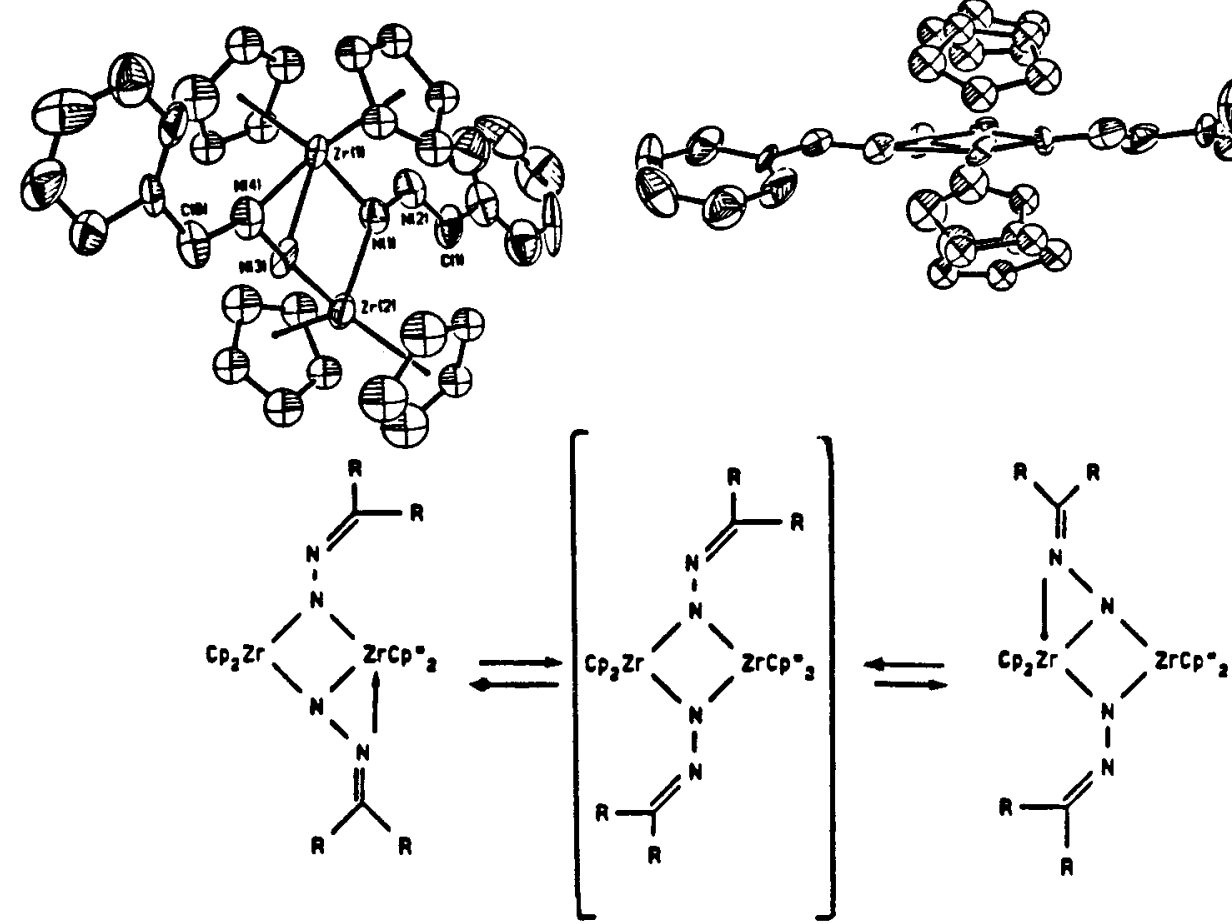

Fig. 1. Structure of the "metalloazine"

units is balanced ultimately by the oxidation of hydrazine to dinitrogen and water. The ease of synthesis of organic starting materials, the availability of the initial organometallic compound and its ready recycle, and the formation of only water and dinitrogen as by-products suggest that this zirconium metalloazine-based route to the synthesis of olefins might constitute practical, new synthetic methodology. There is one problem, however, which still must be overcome before these metalloazines can rightfully claim a place in the repertory of the synthesis-minded organic chemist: competitive formation of mixed azines.

$$
\mathrm{CP}_{2} \mathrm{ZrCl}_{2}+\mathrm{LI}_{2} \mathrm{~N}-\mathrm{N}=\mathrm{C}(\mathrm{H}) \mathrm{R} \rightarrow\left[\mathrm{CP}_{2} \mathrm{Zr}-\mathrm{N}_{1}-\mathrm{N}=\mathrm{C}(\mathrm{H}) \mathrm{R}\right] 2 \underset{2}{\rightleftharpoons} \underset{\mathrm{C}}{\longrightarrow}\left[\mathrm{CP}_{2} \mathrm{Zr}=0\right]_{X}+Y \mathrm{C}(\mathrm{H}) \mathrm{R}+\mathrm{N}_{2}
$$

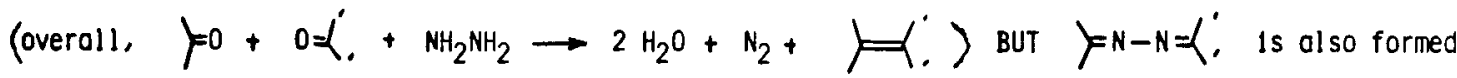

In order to learn to control metalloazine reagent reactivity to favor olefination, a series of trends was investigated. Steric effects were considered, 11 and these results are shown in Table 1. Note that varying the site of alkyl group substitution in cyclohexanones affects relative rates for olefination and azine formation. Substitution at the 2-position gives approximately equimolar amounts of olefin and azine; as the site of substitution moves to the 3-and then 4-positions, the ratio of olefin to azine drops. Thus, substitution remote from the carbonyl has a profound effect on the rate of olefin synthesis but not on that of azine formation. A notable exception is the case in which a methoxy-group is substituted at the 2-position; here the relative rate of olefination vs. azine formation increases. The effect of unsaturation was probed in a second series of experiments.12 Unsaturation favors formation of the olefin, which in some cases becomes the exclusive reaction product. Note, however, that relative rates for olefination do not parallel reduction potentials for the carbonyl compound. Thus, benzaldehyde and less easily reduced cyclohexane carboxaldehyde $\left(E_{1 / 2}=-1.15\right.$ volts vs. -1.85 volts vs. SCE for the analog pentanal ${ }^{13}$ ) react with metalloazine 1 at comparable rates. Therefore, a mechanism involving electron transfer from the complex to the aldehyt or ketone as the rate determining step seems unlikely.

In a third series of experiments ${ }^{12}$ metalloazine 1 was reacted with a series of para-substituted benzaldehyde derivatives. Since each zirconium metalloazine contains two equivalents of reactive groups which are likely transferred sequentially to each of two equivalents of the aldehyde substrate, rate data for these reactions were measured oniy for initial phases of each reaction sequence. In this way, a linear Hammett correlation was obtained for olefination which showed that electron withdrawing groups on the aldehyde increased second order rate constants for olefination. Interestingly, the rate of azine 
Table 1. Reactions of $\left[\mathrm{CP}_{2} \mathrm{Zr}=\mathrm{N}-\mathrm{N}=\mathrm{C}(\mathrm{H})(\mathrm{Ph})\right]_{2}, 1$, with Aldehydes and Ketones

substrate +1 olefina $\frac{\text { ozine }}{(2: E)}$ olefin/azine ounreacted carbony $1^{a}$

Effect of Ring Substitution Patterns<smiles>CC1CCCCC1C</smiles>

30

31

1.00

31

$(1: 1.3)$<smiles>C[C]1CCCC(C)C1</smiles>

27

31

0.87

(1.4:1)

30

42

0.71<smiles>CC1CC(C(C)(C)C)CC[C@@H]1C</smiles><smiles>C/C=C/C</smiles>

Effect of Unsaturation

70

large

Ph<smiles>C[C@H](CCc1ccccc1)Cc1ccccc1</smiles>

THF, $60^{\circ} \mathrm{C}, 9$ hours, ${ }^{\circ} \mathrm{GC}$ yield $( \pm 28)$.

formation appeared to increase with the electron donating ability of the benzaldehyde substituent, suggesting that a strong interaction between the carbonyl group oxygen of the substrate and the zirconium center is important in the transition state leading to formation of azine. In a complementary study, ${ }^{12}$ a series of substituted metalloazine derivatives was reacted with cyclohexanecarboxaldehyde. Reaction rate constants were determined at initial stages of these reactions, too, and a linear Hammett correlation was obtained for olefination which showed that electron donating groups on the metalloazine substituent enhanced rates for olefination. Here, no obvious trend related substituent effects and rates for azine formation.

Based on the latter two studies, we propose that olefin formation occurs by a process of electrophilic attack by the carbonyl group containing substrate upon the metalloazine carbon center of the organometallic in a process in which $C-C$ bond formation is rate determining. Furthermore, we believe that azine formation is initiated by coordination of the carbonyl group of the substrate to the zirconium center. In these two processes steric 

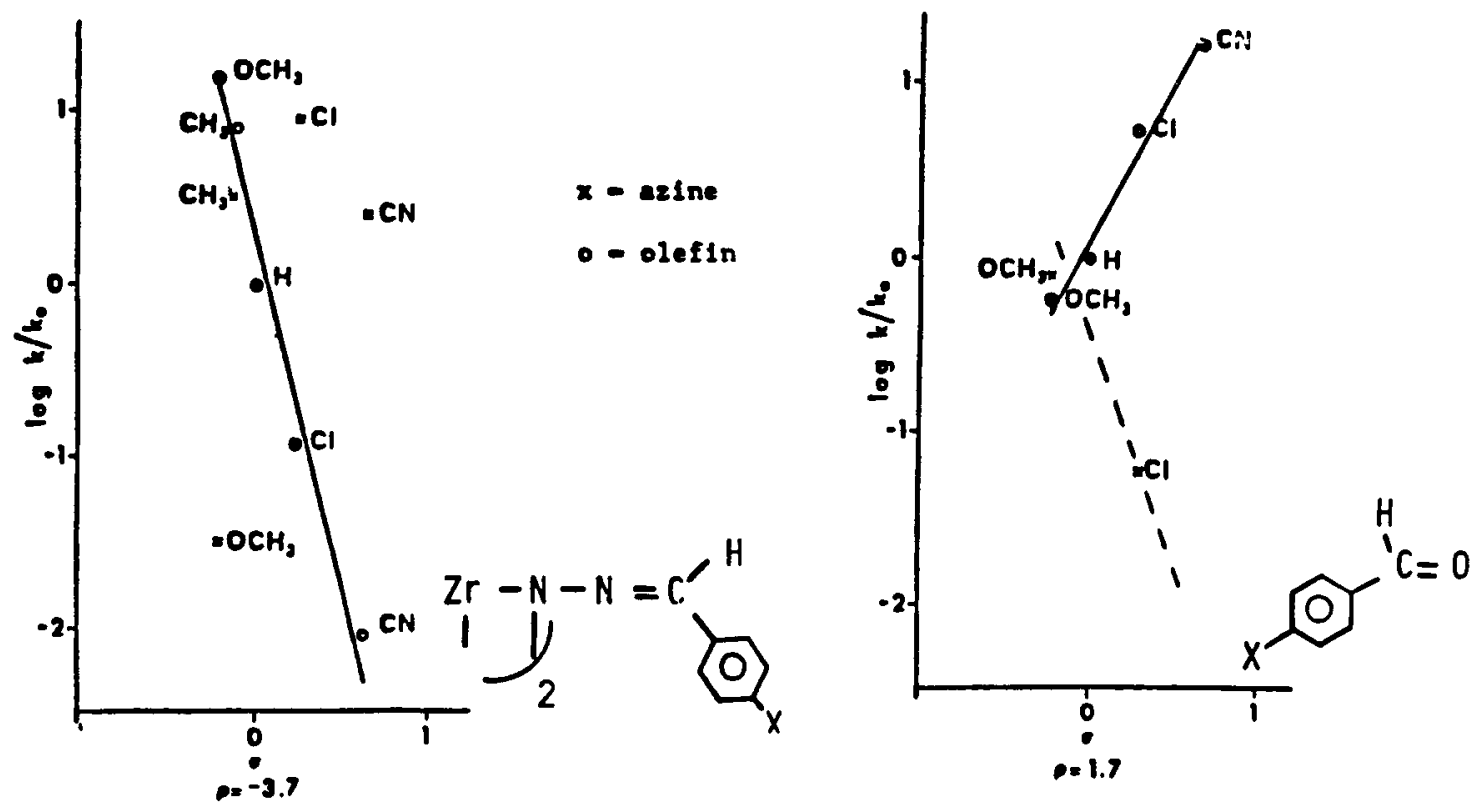

Fig. 2. Olefin vs, azine formation: mechanistic information


Fig. 3 Carbonyl group "anti" approach leads to olefin formation (and explains preference for $Z$ olefin) but coordination to $\mathrm{Zr}$ leads to azine 
effects for positional substitution of the carbonyl compounds would have different effects on rates for olefin vs, azine formation. Consistent with that which was found, substitution at the 2-position of a cyclohexanone should hamper azine formation but should have little effect on olefination since the carbonyl group is likely not coordinated to the zirconium at this stage of the latter reaction sequence. Conversely, substitution at the 4- position should have little effect on azine formation since that substituent is remote from the site of nitrogen atom attack. However, substitution at the 4 - positon would engender unfavorable steric interactions with the ligands on the zirconium center in a transition state in which the carbonyl group and the zirconium atom adapt an antiperiplanar arrangement. In this type of process, however, a zirconium-coordinating substituent on $C-2$ should favor olefination (as was found for 2 -methoxycyclohexanone). It is interesting to note that the $Z$ isomer is the kinetically preferred product of olefination of metalloazines. This is expected for an antiperiplanar transition state in which steric congestion is minimized in the carbon-carbon bond forming sequence. ${ }^{14}$ Rotation about the carbon-carbon bond thus formed, coordination of the "alkoxy" oxygen to zirconium, and elimination of dinitrogen and the "Zr=0" unit yields the $Z$ olefin.

\section{CONCLUSIONS}

We have found that metalloazines can be obtained from readily available organic starting materials and simple organometallic precursors. By reaction of either a diazoalkane or a hydrazone derivative with a metal complex in an appropriate oxidation state, metalloazines of molybdenum and zirconium were prepared. By choice of metal complex with regard to its electronegativity relative to that of carbon, "electrophilic" metalloazines (of molybdenum) or "nucleophilic" ones (of zirconium) could be obtained. These latter species are especially interesting as synthetic reagents because they condense with carbonyl group containing materials to yield olefins in a process in which dinitrogen and water a:e the only sacrificed byproducts of olefination. It remains to determine how variation in ligation on the zirconfum center can be used to enable control of $E, Z$ stereochemistry in the olefination sequence and to suppress formation of azine byproducts.

\section{Acknowledgements}

The authors acknowledge generous support for this research provided by the National Science Foundation and the National Institutes of Health.

\section{REFERENCES}

1. For example see F.N. Tebbe, G.W. Parshall and G.S. Reddy, J. Am. Chem. Soc, 100, $3611-3613$ (1978).

S.H. Pine, R. Zahler, D.A. Evans and R.H. Grubbs, J. Am. Chem. Soc. 102, 3270-3272 (1980).

S.H. Pine, R.J. Pettit, G.D. Gerb, S.H. Cruz, C.H. Gallego, T. Tijerina and R.D. Pine, J. Org. Chem. 50, 1212-1216 (1985).

J.W.S. Stevenson and T.A. Bryson, Tetrahedron Lett., $3143-3146$ (1982).

L. Lombardo, Tetrahedron Lett., 4293.4296 (1982).

J.W. Bruin, G. Schat, O.,S. Akkerman and F. Bickelhaupt, Tetrahedron Lett., 3935-3936, (1980).

J. Hibino, T.Okazoe, K. Takai and H. Nozaki, Tetrahedron Lett., 5579-5580, 5581-5584, (1985).

For a Cr analog, see T. Okazoe,K. Takai and K. Utimoto, J.Am. Chem. Soc. 109, 951.953 (1987)

2. R.R. Schrock, J. Am. Chem. Soc. 98, 5399-5400 (1976).

3. A. Aguero, J. Kress and J.A. Osborn, J. Chem. Soc. Chem. Commun, $531-533$ (1986).

4. F.W. Hartner, Jr. and J. Schwartz, J. Am. Chem. Soc. 103, 4979-4981 (1981).

5. F. W. Hartner, Jr., J. Schwartz and S.M. Clift, J.Am. Chem. Soc. 105, 640-631 (1983)

6. S.M. Clift and J. Schwartz, J, Am. Chem. Soc, 106, 8300 (1984).

7. J.A. Smegel, I.X. Meier and J. Schwartz, J. Am. Chem. Soc. 108, $1322-1323$ (1986).

8. G.M. Arvanitis, J. Schwartz and D. Van Engen, Organometallics $5,2157-2159$ (1986).

9. D. Seebach, Angew. Chem. Int. Ed. Engl. $18,239-258$ (1979).

10. All reactions were run under the same conditions for the same time periods. Products were analyzed by GC. Relative yields of products therefore satisfactorily reflect relative rates for product formation.

11. G.M. Arvanitis, Ph.D. Thesis, Princeton University, 1987

12. G.M. Arvanitis and J. Schwartz, Organometallics $6,421-423$ (1987).

13. L. Meites, CRC Handbook of Series in Organic Electrochemistry, CRC Press: Cleveland, $\mathrm{OH}, 1976$

14. A similar argument has been set forth to explain preferences for the $Z$ olefin in Wittig reactions in which the erythro betaine is formed on condensation of an ylide with a carbonyl compound, and in which collapse of the betaine to olefin is fast. See H.0. House, "Modern Synthetic Reactions", pp. 707-709, W.A. Benjamin, Inc., Menlo Park, CA (1972). 